TRANSACTIONS OF THE

AMERICAN MATHEMATICAL SOCIETY

Volume 362, Number 11, November 2010, Pages 5879-5891

S 0002-9947(2010)04975-X

Article electronically published on June 11, 2010

\title{
THERE IS A VAN DOUWEN MAD FAMILY
}

\author{
DILIP RAGHAVAN
}

\begin{abstract}
We answer a long-standing question of Van Douwen by proving in ZFC that there is a MAD family of functions in $\omega^{\omega}$ that is also maximal with respect to infinite partial functions. In Section 3 we apply the idea of trace introduced in this proof to the still open question of whether analytic MAD families exist in $\omega^{\omega}$. Using the idea of trace, we show that any analytic MAD families that may exist in $\omega^{\omega}$ must satisfy strong combinatorial constraints. We also show that it is consistent to have MAD families in $\omega^{\omega}$ that satisfy these constraints.
\end{abstract}

\section{INTRODUCTION}

The main result of this paper answers a 20 year old question of Eric van Douwen regarding maximal almost disjoint families in Baire space, i.e. $\omega^{\omega}$. Two functions $f$ and $g$ in $\omega^{\omega}$ are said to be almost disjoint if they agree in only finitely many places. Such functions are sometimes also referred to as being eventually different. It is common to identify functions with their graphs. So we adopt the following as our official definition of almost disjointness.

Definition 1.1. Functions $f$ and $g$ in $\omega^{\omega}$ are said to be almost disjoint or a.d. If $|f \cap g|<\omega$. A family $\mathscr{A} \subset \omega^{\omega}$ is said to be a.d. if $\mathscr{A}$ is pairwise a.d., i.e. $\forall f, g \in \mathscr{A}[f \neq g \Longrightarrow|f \cap g|<\omega]$. An a.d. family $\mathscr{A} \subset \omega^{\omega}$ is said to be maximal almost disjoint or $M A D$ if $\forall f \in \omega^{\omega} \exists h \in \mathscr{A}[|h \cap f|=\omega]$.

This notion of a MAD family is closely related to the notion of a MAD family of subsets of $\omega$, even though these notions differ in important ways. Some connections and differences between these two notions have been explored in [10] and [7].

Even though we are primarily interested in MAD families in $\omega^{\omega}$, we will frequently make use of the notion of a MAD family of subsets of a countably infinite set. We fix our terminology in the next definition.

Definition 1.2. Let $X$ be a countably infinite set. $a$ and $b$ in $[X]^{\omega}$ are said to be almost disjoint or a.d. if $|a \cap b|<\omega$. A family $\mathscr{A} \subset[X]^{\omega}$ is said to be a.d. if $\forall a, b \in \mathscr{A}[a \neq b \Longrightarrow|a \cap b|<\omega]$. An a.d. family $\mathscr{A} \subset[X]^{\omega}$ is said to be $M A D$ in $[X]^{\omega}$ if $\forall a \in[X]^{\omega} \exists b \in \mathscr{A}[|a \cap b|=\omega]$.

The above definition departs from the usual practice in that we allow finite subcollections of $[X]^{\omega}$ to count as MAD. Thus if $X=a \cup b$ is a partition of $X$ into two infinite disjoint pieces, then $\{a, b\}$ is MAD in $[X]^{\omega}$ according to our definition.

Received by the editors July 22, 2008.

2010 Mathematics Subject Classification. Primary 03E17, 03E15, 03E05, $03 E 50$.

Key words and phrases. Maximal almost disjoint family, cardinal invariants, analytic set.

The author was partially supported by NSF Grant DMS-0456653.

(C)2010 American Mathematical Society 
We will see below that this non-standard usage allows certain things to be stated in a convenient manner.

Notice that any a.d. family $\mathscr{A} \subset \omega^{\omega}$ is also an a.d. family in $[\omega \times \omega]^{\omega}$, although it can never be a MAD family in $[\omega \times \omega]^{\omega}$ because every function is a.d. from all the vertical columns of $\omega \times \omega$. We are now ready to state Van Douwen's question.

Definition 1.3. $p$ is said to be an infinite partial function if $p$ is a function from some infinite subset of $\omega$ to $\omega$; i.e., for some $X \in[\omega]^{\omega}, p \in \omega^{X}$.

Van Douwen asked whether there is a MAD family of functions $\mathscr{A} \subset \omega^{\omega}$ that is also maximal with respect to infinite partial functions. Let us call such a family a Van Douwen MAD family.

Definition 1.4. An a.d. family $\mathscr{A} \subset \omega^{\omega}$ is called a Van Douwen MAD family if for any infinite partial function $p$ there is $h \in \mathscr{A}$ such that $|h \cap p|=\omega$.

There are several equivalent formulations of Van Douwen's question, and it is instructive to consider some of them. Firstly, observe that $\mathscr{A} \subset \omega^{\omega}$ is a Van Douwen MAD family iff $\mathscr{A} \cup\left\{c_{n}: n \in \omega\right\}$ is MAD in $[\omega \times \omega]^{\omega}$, where $c_{n}$ is the $n^{\text {th }}$ vertical column of $\omega \times \omega$, that is, $c_{n}=\{\langle n, m\rangle: m \in \omega\}$. Another formulation is to ask whether there is an a.d. family $\mathscr{A} \subset \omega^{\omega}$ which is "everywhere maximal" in the following sense. Given an a.d. family $\mathscr{A} \subset \omega^{\omega}$ and a set $X \in[\omega]^{\omega}$, we can consider the restriction of $\mathscr{A}$ to $X, \mathscr{A}\left\lceil X=\{h \uparrow X: h \in \mathscr{A}\}\right.$. This is an a.d. family in $\omega^{X}$. It is easily seen that $\mathscr{A}$ is Van Douwen MAD iff all its restrictions are maximal, that is, $\mathscr{A} \uparrow X$ is MAD in $\omega^{X}$ for all $X \in[\omega]^{\omega}$.

Van Douwen's question dates to the 1980s. It occurs as problem 4.2 in Miller's problem list 9]. In 1999, Zhang [1] obtained some partial results on this problem. He showed that Van Douwen MAD families exist under Martin's Axiom. He also proved that Van Douwen MAD families of various sizes exist in certain forcing extensions.

In Section 2 we solve this problem by proving in ZFC that there is a Van Douwen MAD family of size continuum (Theorem 2.14). The key to our construction is the notion of trace of an a.d. family in $\omega^{\omega}$ introduced in Definitions 2.11 and 2.12 . We will rephrase Van Douwen's problem in terms of this notion: Van Douwen MAD families are those a.d. families that have "large trace". We will make use of certain combinatorial properties of the cardinal invariant non $(\mathcal{M})$ to construct such a family with a sufficiently "large trace".

In Section 3 we will show that this concept of trace is also useful for analyzing the still open problem of whether there is an analytic MAD family in $\omega^{\omega}$. This question is one way to make precise the intuitive question: "Does there exist a concrete example of a MAD family in $\omega^{\omega}$ ?", which naturally arises as MAD families are constructed using the axiom of choice. By a classical result of Mathias 8], there are no analytic MAD families in $[\omega]^{\omega}$. However the corresponding question for $\omega^{\omega}$ remains open despite several attempts (see [6]). In Section 3, we will use the notion of trace to show that any analytic MAD families that may exist in $\omega^{\omega}$ must satisfy certain strong combinatorial constraints (Theorems 3.2, 3.20 and 3.25). These results improve the result of Steprāns [ 6 , that strongly MAD families cannot be analytic. But we will also show that it is consistent with ZFC to have MAD families in $\omega^{\omega}$ that satisfy these combinatorial constraints; so these constraints by themselves do not preclude the existence of an analytic MAD family in $\omega^{\omega}$. 


\section{A VAn Douwen MAD family In ZFC}

In this section we will prove in ZFC that there is a Van Douwen MAD family of size Continuum. The starting point for our construction is the following wellknown characterization of the cardinal non $(\mathcal{M})$, due to Bartoszyński. The reader may consult [1] or [2] for a proof of this.

Definition 2.1. non $(\mathcal{M})$ is the least size of a non-meager set of reals.

Definition 2.2. Let $h \in \omega^{\omega}$ be such that $\forall n \in \omega[h(n) \geq 1]$. An $h$-slalom is a function $S: \omega \rightarrow[\omega]^{<\omega}$ such that for all $n \in \omega,|S(n)| \leq h(n)$.

Theorem 2.3 (Bartoszyński [1]). Let $\kappa$ be an infinite cardinal. The following are equivalent:

(1) Every set of reals of size less than $\kappa$ is meager.

(2) For every family $F \subset \omega^{\omega}$ with $|F|<\kappa$, there is an infinite partial function $g$ from $\omega$ to $\omega$ such that $\forall f \in F[|f \cap g|<\omega]$.

(3) For every $h$ and for every family of $h$-slaloms $F$ with $|F|<\kappa$, there is a $g \in \omega^{\omega}$ such that $\forall S \in F \forall^{\infty} n \in \omega[g(n) \notin S(n)]$.

Our first task is to strengthen condition (3) above. We will first show that if $F$ is a family of $h$-slaloms of size less than non $(\mathcal{M})$, then we can get a one-to-one function $g$, which is eventually outside all the slaloms in $F$ (Lemma 2.4). We will then show that we can, in fact, get a suitably "wide" slalom which is eventually disjoint from all slaloms in $F$ (Lemma 2.6). Lemma 2.4 was independently discovered and used by Brendle, Spinas and Zhang [5].

Lemma 2.4. Let $\kappa=\operatorname{non}(\mathcal{M})$ and let $F$ be a family of h-slaloms with $|F|<\kappa$. There is a one-to-one function $g \in \omega^{\omega}$ such that $\forall S \in F \forall^{\infty} n \in \omega[g(n) \notin S(n)]$.

Proof. Our proof is similar to the argument in Bartoszyński [1. Write $F=\left\langle S_{\xi}\right.$ : $\xi<\lambda\rangle$, where $\lambda=|F|$. Define a new function $h^{\prime}$ and a family of $h^{\prime}$-slaloms as follows:

$$
\begin{aligned}
h^{\prime}(n) & =\sum_{i \leq n} h(i), \\
\forall \xi<\lambda S_{\xi}^{\prime}(n) & =\bigcup_{i \leq n} S_{\xi}(i) .
\end{aligned}
$$

Clearly, $\left\langle S_{\xi}^{\prime}: \xi<\lambda\right\rangle$ is a family of $h^{\prime}$-slaloms. Now, for each $i \in \omega$, let $T_{i}: \omega \rightarrow$ $[\omega]^{<\omega}$ be defined by $T_{i}(n)=\{i\}$. It is clear that $\left\langle S_{\xi}^{\prime}: \xi\langle\lambda\rangle \cup\left\langle T_{i}: i \in \omega\right\rangle\right.$ is a family of fewer than $\kappa h^{\prime}$-slaloms. Thus by (3) of Theorem 2.3 we can choose $g \in \omega^{\omega}$ such that the following hold:

(1) $\forall \xi<\lambda \forall^{\infty} n \in \omega\left[g(n) \notin S_{\xi}^{\prime}(n)\right]$,

(2) $\forall i \in \omega \forall^{\infty} n \in \omega\left[g(n) \notin T_{i}(n)\right]$.

Property 2 implies that $g$ takes any given value only finitely often. Thus we may choose a one-to-one infinite partial function $g^{\prime} \subset g$. Let $X=\operatorname{dom}\left(g^{\prime}\right)$. By property (1) we obviously have that for any $\xi<\lambda, \forall^{\infty} n \in \omega\left[n \in X \Longrightarrow g^{\prime}(n) \notin S_{\xi}^{\prime}(n)\right]$. Let $\left\langle x_{n}: n \in \omega\right\rangle$ be the increasing enumeration of $X$. For $n \in \omega$, set $g^{\prime \prime}(n)=$ $g^{\prime}\left(x_{n}\right)$. Since $g^{\prime}$ is one-to-one, $g^{\prime \prime}$ is also one-to-one. We claim that $g^{\prime \prime}$ is the function we are looking for. Indeed, fix $\xi<\lambda$. We know that $\exists m \in \omega \forall n \geq$ 
$m\left[n \in X \Longrightarrow g^{\prime}(n) \notin S_{\xi}^{\prime}(n)\right]$. We will show that $\forall n \geq m\left[g^{\prime \prime}(n) \notin S_{\xi}(n)\right]$. Suppose, for a contradiction, that $g^{\prime \prime}(n)=g^{\prime}\left(x_{n}\right) \in S_{\xi}(n)$, for some $n \geq m$. Note that we have $m \leq n \leq x_{n}$. Thus, by the definition of $S_{\xi}^{\prime}, S_{\xi}(n) \subset S_{\xi}^{\prime}\left(x_{n}\right)$. Therefore, we have that $g^{\prime}\left(x_{n}\right) \in S_{\xi}^{\prime}\left(x_{n}\right)$. But this is a contradiction because $x_{n} \geq m$ and $x_{n} \in X$.

Convention 2.5. In what follows we will only be concerned with $h$-slaloms for the function $h(n)=2^{n}$. We will simply refer to these as slaloms, suppressing mention of $h$.

Lemma 2.6. Let $F=\left\langle S_{\xi}: \xi<\lambda\right\rangle$ be a family of slaloms with $\lambda<\operatorname{non}(\mathcal{M})$. There is a slalom $S$ such that $\forall n \in \omega\left[|S(n)|=2^{n}\right]$ and $\forall \xi<\lambda \forall^{\infty} n \in \omega\left[S(n) \cap S_{\xi}(n)=0\right]$.

Proof. For all $n \in \omega$ set $l_{n}=2^{n}-1$ and $I_{n}=\left[l_{n}, l_{n+1}\right)$. For each $\xi<\lambda$ define $S_{\xi}^{\prime}$ by stipulating that $\forall k, n \in \omega\left[S_{\xi}^{\prime}(k)=S_{\xi}(n)\right.$ iff $\left.k \in I_{n}\right]$. We have that for all $k \in \omega,\left|S_{\xi}^{\prime}(k)\right| \leq\left|S_{\xi}(n)\right| \leq 2^{n}$, where $k \in I_{n}$. But if $k \in I_{n}$, then $2^{n} \leq 2^{k}$ and so $\left|S_{\xi}^{\prime}(k)\right| \leq 2^{k}$. Therefore, $\left\langle S_{\xi}^{\prime}: \xi<\lambda\right\rangle$ is a family of fewer than non $(\mathcal{M})$ many slaloms. By applying Lemma 2.4 we can find a one-to-one function $g \in \omega^{\omega}$ such that for every $\xi<\lambda, \forall^{\infty} k \in \omega\left[g(k) \notin S_{\xi}^{\prime}(k)\right]$. Now define $S$ by setting $S(n)=\left\{g(k): k \in I_{n}\right\}$. Since $g$ is one-to-one, $|S(n)|=\left|I_{n}\right|=2^{n}$. Fix $\xi<\lambda$. We know that $\exists m \in \omega \forall k \geq m\left[g(k) \notin S_{\xi}^{\prime}(k)\right]$. We claim that for any $n \geq m$, $S(n) \cap S_{\xi}(n)=0$. Suppose to the contrary that for some $n \geq m, g(k) \in S_{\xi}(n)$ for some $k \in I_{n}$. Then since $k \in I_{n}, S_{\xi}^{\prime}(k)=S_{\xi}(n)$, and so we get that $g(k) \in S_{\xi}^{\prime}(k)$. But this is a contradiction because $m \leq n \leq l_{n} \leq k$.

Lemma 2.7. Let $S$ be a slalom such that $\forall n \in \omega\left[|S(n)|=2^{n}\right]$. There exists an a.d. family $\mathscr{A} \subset \omega^{\omega}$ such that $|\mathscr{A}|=\mathfrak{c}$ and for every $f \in \mathscr{A}, \forall n \in \omega[f(n) \in S(n)]$.

Proof. Since $|S(n)|=\left|{ }^{n} 2\right|$, we can assign to each $\sigma \in{ }^{n} 2$ a unique number $k_{\sigma} \in S(n)$. Now, for each $\mu \in 2^{\omega}$, define $f_{\mu} \in \omega^{\omega}$ by setting $f_{\mu}(n)=k_{\mu \nmid n} \in S(n)$. Suppose $\mu \neq \nu \in 2^{\omega}$. Then there is $m \in \omega$ such that $\mu(m) \neq \nu(m)$. So for all $n>m$, $\mu\left\lceil n \neq \nu \uparrow n\right.$, and so $f_{\mu}(n)=k_{\mu\lceil n} \neq k_{\nu\lceil n}=f_{\nu}(n)$. Thus $\mathscr{A}=\left\{f_{\mu}: \mu \in 2^{\omega}\right\}$ is as required.

Definition 2.8. Let $A, B \subset \omega^{\omega}$ be two families of functions. We will write $A \perp B$ to mean that $\forall f \in A \forall g \in B[|f \cap g|<\omega]$

The next lemma will play an important role in our construction. The proof of this lemma will use Lemma 2.7 and is the reason why we set out to strengthen clause (3) of Theorem 2.3 .

Lemma 2.9. Let $\kappa=\operatorname{non}(\mathcal{M})$. Let $F=\left\langle f_{\alpha}: \alpha<\kappa\right\rangle \subset \omega^{\omega}$. There is a sequence $\left\langle\mathscr{A}_{\alpha}: \alpha<\kappa\right\rangle$ such that the following hold:

(1) $\mathscr{A}_{\alpha} \subset \omega^{\omega}$ is an a.d. family.

(2) $\left|\mathscr{A}_{\alpha}\right|=\mathfrak{c}$.

(3) For all $\beta<\alpha<\kappa, \mathscr{A}_{\alpha} \perp \mathscr{A}_{\beta}$.

(4) $\mathscr{A}_{\alpha} \perp\left\{f_{\beta}: \beta \leq \alpha\right\}$.

Proof. We will construct the family $\left\langle\mathscr{A}_{\alpha}: \alpha<\kappa\right\rangle$ by induction. We will simultaneously build a family of slaloms $\left\langle S_{\alpha}: \alpha<\kappa\right\rangle$ and ensure that for all $\alpha<\kappa$, 
$\forall f \in \mathscr{A}_{\alpha} \forall n \in \omega\left[f(n) \in S_{\alpha}(n)\right]$. Fix $\alpha<\kappa$ and suppose that $\left\langle\mathscr{A}_{\beta}: \beta<\alpha\right\rangle$ and $\left\langle S_{\beta}: \beta<\alpha\right\rangle$ are already given to us. For each $\beta \leq \alpha$, define a slalom $T_{\beta}$ by $T_{\beta}(n)=\left\{f_{\beta}(n)\right\}$. Thus, $\left\{S_{\beta}: \beta<\alpha\right\} \cup\left\{T_{\beta}: \beta \leq \alpha\right\}$ is a family of fewer than $\kappa$ slaloms. So we can apply Lemma 2.6 to find a slalom $S_{\alpha}$ such that the following hold:

(a) $\forall n \in \omega\left[\left|S_{\alpha}(n)\right|=2^{n}\right]$.

(b) $\forall \beta<\alpha \forall^{\infty} n \in \omega\left[S_{\alpha}(n) \cap S_{\beta}(n)=0\right]$.

(c) $\forall \beta \leq \alpha \forall^{\infty} n \in \omega\left[S_{\alpha}(n) \cap T_{\beta}(n)=0\right]$.

Property (a) allows us to apply Lemma 2.7 to $S_{\alpha}$ to find an a.d. family $\mathscr{A}_{\alpha} \subset \omega^{\omega}$ with $\left|\mathscr{A}_{\alpha}\right|=\mathfrak{c}$ and with the property that $\forall f \in \mathscr{A}_{\alpha} \forall n \in \omega\left[f(n) \in S_{\alpha}(n)\right]$. Thus $\mathscr{A}_{\alpha}$ satisfies requirements (1) and (2). We will check requirements (3) and (4). Fix $f \in \mathscr{A}_{\alpha}$ and $g \in \mathscr{A}_{\beta}$ for some $\beta<\alpha$. We know that there is $m \in \omega$ such that $\forall n \geq m\left[S_{\alpha}(n) \cap S_{\beta}(n)=0\right]$. Since $\forall n \in \omega\left[f(n) \in S_{\alpha}(n) \wedge g(n) \in S_{\beta}(n)\right]$, it follows that $\forall n \geq m[f(n) \neq g(n)]$. To verify (4), fix $f \in \mathscr{A}_{\alpha}$ and some $\beta \leq \alpha$. Again we know that there is $m \in \omega$ such that $\forall n \geq m\left[S_{\alpha}(n) \cap\left\{f_{\beta}(n)\right\}=0\right]$ and that $\forall n \in \omega\left[f(n) \in S_{\alpha}(n)\right]$. Therefore, it follows that $\forall n \geq m\left[f(n) \neq f_{\beta}(n)\right]$.

We are now ready to construct our Van Douwen MAD family. In order to ensure that our family is Van Douwen MAD we will introduce the notion of the trace of an a.d. family. The idea is that if an a.d. family has a "sufficiently large" trace, then it must be Van Douwen MAD.

Convention 2.10. By Theorem 2.3 there is a family $F=\left\langle f_{\alpha}: \alpha<\operatorname{non}(\mathcal{M})\right\rangle \subset \omega^{\omega}$ such that for every infinite partial function $g$ there is an $\alpha<\operatorname{non}(\mathcal{M})$ such that $\left|g \cap f_{\alpha}\right|=\omega$. For the remainder of this section let us fix such a family $F$.

Definition 2.11. Let $\mathscr{A} \subset \omega^{\omega}$ be an a.d. family and let $f \in \omega^{\omega}$. We define $\mathscr{A} \cap f=\{f \cap h: h \in \mathscr{A} \wedge|f \cap h|=\omega\}$. Note that this is an a.d. family in $[f]^{\omega}$.

Definition 2.12. Let $\mathscr{A} \subset \omega^{\omega}$ be an a.d. family. The trace of $\mathscr{A}$, written $\operatorname{tr}(\mathscr{A})$, is $\left\{f \in \omega^{\omega}: \mathscr{A} \cap f\right.$ is a MAD family in $\left.[f]^{\omega}\right\}$.

The reader might wonder why we define the trace of $\mathscr{A}$ by considering $\mathscr{A} \cap f$ only for total functions $f$, and not for all infinite partial functions. It will become clear later (see Lemma 3.9) that this restriction actually makes no difference.

Lemma 2.13. Let $\mathscr{A} \subset \omega^{\omega}$ be an a.d. family such that $F \subset \operatorname{tr}(\mathscr{A})$. Then $\mathscr{A}$ is Van Douwen MAD.

Proof. Indeed, let $g$ be an infinite partial function. By the definition of $F$, there is $\alpha<\operatorname{non}(\mathcal{M})$ such that $\left|g \cap f_{\alpha}\right|=\omega$. Since $F \subset \operatorname{tr}(\mathscr{A}), \mathscr{A} \cap f_{\alpha}$ is a MAD family on $f_{\alpha}$. So there is $h \in \mathscr{A}$ such that $h \cap f_{\alpha}$ meets $g \cap f_{\alpha}$ in an infinite set, whence we get that $|h \cap g|=\omega$.

Theorem 2.14. There is a Van Douwen MAD family of size $\mathfrak{c}$.

Proof. In view of Lemma 2.13, it is enough to construct an a.d. family $\mathscr{A}$ of size $\mathfrak{c}$ such that $F \subset \operatorname{tr}(\mathscr{A})$. We will use Lemma 2.9 to do this. Fix a sequence $\left\langle\mathscr{A}_{\alpha}: \alpha<\right.$ $\operatorname{non}(\mathcal{M})\rangle$ as in Lemma 2.9, $\mathscr{A}$ will be constructed as the union of an increasing sequence of a.d. families. Thus, we will construct a sequence $\left\langle\mathscr{C}_{\alpha}: \alpha<\operatorname{non}(\mathcal{M})\right\rangle$ such that:

(1) $\mathscr{C}_{\alpha} \subset \omega^{\omega}$ is an a.d. family,

(2) $\forall \beta<\alpha<\operatorname{non}(\mathcal{M})\left[\mathscr{C}_{\beta} \subset \mathscr{C}_{\alpha}\right]$, 
(3) $f_{\alpha} \in \operatorname{tr}\left(\mathscr{C}_{\alpha}\right)$,

(4) $\forall h \in \mathscr{C}_{\alpha} \exists \beta \leq \alpha \exists g \in \mathscr{A}_{\beta} \exists X \in[\omega]^{\omega}\left[h=\left(f_{\beta}\lceil X) \cup(g\lceil(\omega \backslash X))]\right.\right.$,

(5) $\left|\mathscr{C}_{0}\right|=\mathfrak{c}$

To construct $\mathscr{C}_{0}$, we fix a MAD family $\left\{a_{\xi}: \xi<\mathfrak{c}\right\}$ on $\omega$. Put $\mathscr{A}_{0}=\left\{g_{\xi}: \xi<\mathfrak{c}\right\}$. For each $\xi<\mathfrak{c}$, let $h_{\xi}=\left(f_{0}\left\lceil a_{\xi}\right) \cup\left(g_{\xi}\left\lceil\left(\omega \backslash a_{\xi}\right)\right)\right.\right.$, and put $\mathscr{C}_{0}=\left\{h_{\xi}: \xi<\mathfrak{c}\right\}$. We will check that $\mathscr{C}_{0}$ is a.d. Indeed, if $\xi_{0}<\xi_{1}$, then since $a_{\xi_{0}} \cap a_{\xi_{1}}$ is finite, $\mid f_{0}\left\lceil a_{\xi_{0}} \cap f_{0}\left\lceil a_{\xi_{1}} \mid<\omega\right.\right.$. Next, since $\mathscr{A}_{0} \perp\left\{f_{0}\right\}$, we have that both $\left(f_{0}\left\lceil a_{\xi_{0}}\right) \cap\right.$ $\left(g_{\xi_{1}}\left\lceil\left(\omega \backslash a_{\xi_{1}}\right)\right)\right.$ and $\left(f_{0}\left\lceil a_{\xi_{1}}\right) \cap\left(g_{\xi_{0}}\left\lceil\left(\omega \backslash a_{\xi_{0}}\right)\right)\right.\right.$ are finite. Finally, since $\mathscr{A}_{0}$ is an a.d. family, we know that $\mid g_{\xi_{0}}\left\lceil\left(\omega \backslash a_{\xi_{0}}\right) \cap g_{\xi_{1}} \uparrow\left(\omega \backslash a_{\xi_{1}}\right) \mid<\omega\right.$. Thus, we conclude that $\left|h_{\xi_{0}} \cap h_{\xi_{1}}\right|<\omega$. Next, it is clear from the construction that $f_{0} \in \operatorname{tr}\left(\mathscr{C}_{0}\right)$, and that $\mathscr{C}_{0}$ satisfies clauses (4) and (5).

To continue the construction, suppose that we are given the sequence $\left\langle\mathscr{C}_{\beta}: \beta<\right.$ $\alpha\rangle$. Set $\mathscr{C}=\bigcup_{\beta<\alpha} \mathscr{C}_{\beta}$ and consider $\mathscr{C} \cap f_{\alpha}$. This is an a.d. family on $f_{\alpha}$. If it is a MAD family (either finite or infinite), then $f_{\alpha}$ is already in $\operatorname{tr}(\mathscr{C})$, and there is nothing more to be done. In this case, we set $\mathscr{C}_{\alpha}=\mathscr{C}$. So, say that $\mathscr{C} \cap f_{\alpha}$ is not MAD. We can extend it to a MAD family, say $\mathscr{B}$, on $f_{\alpha}$. Consider the family $\left\{Y \in[\omega]^{\omega}: f_{\alpha}\left\lceil Y \in \mathscr{B} \backslash\left(\mathscr{C} \cap f_{\alpha}\right)\right\}\right.$. Note that this is an a.d. family on $\omega$. We may assume WLOG that it has size $\mathfrak{c}$. Let $\left\{a_{\xi}: \xi<\mathfrak{c}\right\}$ enumerate this family. Put $\mathscr{A}_{\alpha}=\left\{g_{\xi}: \xi<\mathfrak{c}\right\}$. For each $\xi<\mathfrak{c}$, set $h_{\xi}=\left(f_{\alpha}\left\lceil a_{\xi}\right) \cup\left(g_{\xi}\left\lceil\left(\omega \backslash a_{\xi}\right)\right)\right.\right.$, and put $\mathscr{D}=\left\{h_{\xi}: \xi<\mathfrak{c}\right\}$. It is easily argued, as for $\mathscr{C}_{0}$, that $\mathscr{D}$ is a.d. We will check that $\mathscr{C} \perp \mathscr{D}$. Fix $h \in \mathscr{C}$ and $\xi<\mathfrak{c}$. If $h \cap f_{\alpha}$ is finite, then so is $h \cap f_{\alpha}\left\lceil a_{\xi}\right.$. On the other hand, if $h \cap f_{\alpha}$ is infinite, then $h \cap f_{\alpha} \in \mathscr{C} \cap f_{\alpha}$. But then $\mid f_{\alpha}\left\lceil a_{\xi} \cap h \mid<\omega\right.$ because $\mathscr{B}$ is an a.d. family. Thus in either case, $h \cap f_{\alpha}\left\lceil a_{\xi}\right.$ is finite. To deal with $h \cap g_{\xi} \uparrow\left(\omega \backslash a_{\xi}\right)$, by clause (4), we know that for some $\gamma \leq \beta<\alpha, h=\left(f_{\gamma} \uparrow X\right) \cup(g \uparrow(\omega \backslash X))$, where $X \in[\omega]^{\omega}$ and $g \in \mathscr{A}_{\gamma}$. But since $\mathscr{A}_{\alpha} \perp\left\{f_{\gamma}\right\}, \mid\left(f_{\gamma}\lceil X) \cap\left(g_{\xi} \uparrow\left(\omega \backslash a_{\xi}\right)\right) \mid<\omega\right.$, and since $\mathscr{A}_{\alpha} \perp \mathscr{A}_{\gamma}$, we know that $\mid\left(g_{\xi}\left\lceil\left(\omega \backslash a_{\xi}\right)\right) \cap\left(g\lceil(\omega \backslash X)) \mid<\omega\right.\right.$. Therefore, $h \cap g_{\xi} \uparrow\left(\omega \backslash a_{\xi}\right)$ is also finite, and so $\left|h \cap h_{\xi}\right|<\omega$. Hence, we can define $\mathscr{C}_{\alpha}=\mathscr{C} \cup \mathscr{D}$.

Now, it is clear that $\mathscr{C}_{\alpha}$ satisfies clauses (1), (2) and (4). We just need to verify that $f_{\alpha} \in \operatorname{tr}\left(\mathscr{C}_{\alpha}\right)$. So we need to check that $\mathscr{C}_{\alpha} \cap f_{\alpha}$ is a MAD family on $f_{\alpha}$. But clearly $\mathscr{C}_{\alpha} \cap f_{\alpha}=\left(\mathscr{C} \cap f_{\alpha}\right) \cup\left(\mathscr{D} \cap f_{\alpha}\right)$. Fix $X \in[\omega]^{\omega}$. Since $\mathscr{B}$ is a MAD family on $f_{\alpha}$, there is $Y \in[\omega]^{\omega}$ such that $f_{\alpha}\left\lceil Y \in \mathscr{B}\right.$ and $\mid f_{\alpha}\left\lceil X \cap f_{\alpha}\lceil Y \mid=\omega\right.$. If $f_{\alpha} \mid Y \in \mathscr{C} \cap f_{\alpha}$, then we are done. If it is not, then $Y=a_{\xi}$ for some $\xi<\mathfrak{c}$. It follows that $\mid f_{\alpha}\left\lceil X \cap h_{\xi} \mid=\omega\right.$. But since $h_{\xi} \in \mathscr{D}$, we are done.

Definition 2.15. Let $\mathfrak{a}_{\mathfrak{v}}$ denote the least size of a Van Douwen MAD family. By Theorem 2.14, this cardinal is well defined. Let $\mathfrak{a}_{\mathfrak{e}}$ denote the least size of a MAD family of functions in $\omega^{\omega}$.

Since any Van Douwen MAD family is MAD, we have $\mathfrak{a}_{\mathfrak{e}} \leq \mathfrak{a}_{\mathfrak{v}}$.

Question 2.16. Is it consistent to have $\mathfrak{a}_{\mathfrak{e}}<\mathfrak{a}_{\mathfrak{v}}$ ?

\section{Definability of MAD families in $\omega^{\omega}$}

In this section we will apply the notion of trace introduced above to the problem of whether there are any analytic MAD families in $\omega^{\omega}$. It is a well-known result of Mathias $\left[8\right.$ that there are no analytic MAD families in $[\omega]^{\omega}$. Now, if $\mathscr{A} \subset \omega^{\omega}$ is Van Douwen MAD, then $\mathscr{A} \cup\left\{c_{n}: n \in \omega\right\}$ is a MAD family in $[\omega \times \omega]^{\omega}$, where $c_{n}$ denotes the $n^{\text {th }}$ vertical column of $\omega \times \omega$. It follows that there are no analytic Van Douwen MAD families in $\omega^{\omega}$. We will show below (Theorem 3.2) that analytic 
a.d. families must be the "opposite" of Van Douwen MAD families in the following sense. Van Douwen MAD families are those a.d. families whose trace is as large as possible; on the other hand, the trace of an analytic a.d. family must be as small as possible. We will then show that it is consistent to have MAD families in $\omega^{\omega}$ whose trace is as small as possible (Theorem 3.14). Then we define some ideals on $\omega$ that measure how far away a given MAD family in $\omega^{\omega}$ is from being Van Douwen MAD, and we show that these ideals are large for analytic MAD families (Theorem 3.25).

Definition 3.1. Let $\mathscr{A} \subset[\omega \times \omega]^{\omega}$ be an a.d. family and let $X \in[\omega \times \omega]^{\omega}$. We say that $X$ avoids $\mathscr{A}$ if for any finite collection $\left\{h_{0}, \ldots, h_{n}\right\} \subset \mathscr{A},\left|X \backslash h_{0} \cup \cdots \cup h_{n}\right|=$ $\omega$.

Theorem 3.2. Let $\mathscr{A} \subset \omega^{\omega}$ be an a.d. family and let $X \in[\omega \times \omega]^{\omega}$ avoid $\mathscr{A}$. Suppose that $\mathscr{A}$ is analytic in $\omega^{\omega}$. There is $Y \in[X]^{\omega}$ such that $\forall h \in \mathscr{A}[|h \cap Y|<\omega]$.

Proof. This follows easily from the result of Mathias 8 that there are no analytic MAD families in $[\omega]^{\omega}$. To see this, give the space $2^{X}$ the Tychonoff product topology, with 2 having the discrete topology. Since $X$ is a countable set, this is homeomorphic to $2^{\omega}$ with the usual topology. Define a map $\Psi: \omega^{\omega} \rightarrow 2^{X}$ by stipulating that $\forall\langle n, m\rangle \in X[\Psi(f)(\langle n, m\rangle)=1 \leftrightarrow\langle n, m\rangle \in f]$. Thus $\Psi(f)$ is the characteristic function of $X \cap f$. This map is continuous. It follows from this that $\mathscr{A} \cap X=\{h \cap X: h \in \mathscr{A} \wedge|h \cap X|=\omega\}$ is analytic. Now, $\mathscr{A} \cap X$ is an a.d. family on $X$. By a theorem of Mathias [8] we know that there are no analytic MAD families on $X$. Therefore, if $\mathscr{A} \cap X$ is infinite, it is not MAD on $X$, and we will get the conclusion of the theorem. On the other hand, if $\mathscr{A} \cap X$ is finite, then since $X$ avoids $\mathscr{A}, Y=X \backslash \bigcup(\mathscr{A} \cap X)$ will satisfy the conclusion of the theorem. Hence, either way, the theorem is proved.

Definition 3.3. An a.d. family $\mathscr{A} \subset \omega^{\omega}$ is said to have trivial trace if no member of $\operatorname{tr}(\mathscr{A})$ avoids $\mathscr{A}$.

Corollary 3.4. Suppose $\mathscr{A} \subset \omega^{\omega}$ is an analytic a.d. family. Then $\mathscr{A}$ has trivial trace.

Proof. If $f$ is a member of $\operatorname{tr}(\mathscr{A})$ which avoids $\mathscr{A}$, then putting $f=X$ in Theorem 3.2 will give a contradiction.

Corollary 3.5. There are no analytic Van Douwen MAD families in $\omega^{\omega}$.

Steprāns [6] introduced the notion of a strongly MAD family and proved that they can't be analytic.

Definition 3.6. A MAD family $\mathscr{A} \subset \omega^{\omega}$ is strongly $M A D$ if for every collection $\left\{f_{i}: i \in \omega\right\} \subset \omega^{\omega}$ where each $f_{i}$ avoids $\mathscr{A}$, there is an $h \in \mathscr{A}$ such that $\forall i \in$ $\omega\left[\left|f_{i} \cap h\right|=\omega\right]$.

Lemma 3.7. Let $\mathscr{A} \subset \omega^{\omega}$ be strongly MAD. Let $\left\{g_{i}: i \in \omega\right\}$ be a collection of infinite partial functions from $\omega$ to $\omega$ such that each $g_{i}$ avoids $\mathscr{A}$. There is an $h \in \mathscr{A}$ such that $\forall i \in \omega\left[\left|h \cap g_{i}\right|=\omega\right]$. In particular, strongly MAD families are Van Douwen MAD.

Proof. Let $h_{0} \neq h_{1}$ be two distinct members of $\mathscr{A}$. For each $i \in \omega$, let $a_{i}=\operatorname{dom}\left(g_{i}\right)$ and let $b_{i}=\omega \backslash a_{i}$. For each $i \in \omega$, define $f_{i}^{0}=g_{i} \cup h_{0}\left\lceil b_{i}\right.$ and $f_{i}^{1}=g_{i} \cup h_{1}\left\lceil b_{i}\right.$. Since $g_{i}$ avoids $\mathscr{A}$, both $f_{i}^{0}$ and $f_{i}^{1}$ avoid $\mathscr{A}$. Thus $\left\{f_{i}^{j}: i \in \omega \wedge j \in 2\right\}$ is a 
countable collection of total functions avoiding $\mathscr{A}$. So we may choose $h \in \mathscr{A}$ such that $\forall i \in \omega \forall j \in 2\left[\left|h \cap f_{i}^{j}\right|=\omega\right]$. We will show that $\forall i \in \omega\left[\left|g_{i} \cap h\right|=\omega\right]$. If $g_{i} \cap h$ is finite, then since both $f_{i}^{0} \cap h$ and $f_{i}^{1} \cap h$ are infinite, it follows that $\left|h_{0} \cap h\right|=\omega$ and that $\left|h_{1} \cap h\right|=\omega$. But since $\mathscr{A}$ is an a.d. family this means that $h=h_{0}$ and $h=h_{1}$, which is a contradiction.

Corollary 3.8 (Steprāns [6]). There are no analytic strongly MAD families in $\omega^{\omega}$.

Corollary 3.5 is strictly stronger than Corollary 3.8. It is easy to modify the construction in Theorem 2.14 to ensure that the Van Douwen MAD family constructed there is not strongly MAD.

It is an open problem whether there are any analytic MAD families in $\omega^{\omega}$. In fact, it is not even known if a MAD family in $\omega^{\omega}$ can be closed. Since Theorem 3.2 puts a strong restriction on such MAD families, one might conjecture that there are no MAD families that satisfy the conclusion of Theorem 3.2 at all. However, we will show below that this is consistently false. We will first argue that it is sufficient to build a MAD family with a trivial trace.

Lemma 3.9. Let $\mathscr{A} \subset \omega^{\omega}$ be a MAD family with a trivial trace. Suppose $X \in$ $[\omega \times \omega]^{\omega}$ avoids $\mathscr{A}$. There is $Y \in[X]^{\omega}$ such that $\forall h \in \mathscr{A}[|h \cap Y|<\omega]$.

Proof. Let $\mathscr{A} \cap X=\{h \cap X: h \in \mathscr{A} \wedge|h \cap X|=\omega\}$. If $\mathscr{A} \cap X$ is finite, then since $X$ avoids $\mathscr{A}, Y=X \backslash \bigcup(\mathscr{A} \cap X)$ will be as required. So assume that $\mathscr{A} \cap X$ is infinite. Choose a countably infinite collection $\left\{h_{i}: i \in \omega\right\} \subset \mathscr{A}$ such that $\left|h_{i} \cap X\right|=\omega$ for each $i$, and put $p_{i}=h_{i} \cap X$. Thus $\left\{p_{i}: i \in \omega\right\}$ forms an a.d. family of infinite partial functions. We may choose infinite partial functions $g_{i} \subset p_{i}$ such that $\forall i<j<\omega\left[\operatorname{dom}\left(g_{i}\right) \cap \operatorname{dom}\left(g_{j}\right)=0\right]$. Now if we put $g=\bigcup g_{i}$, then $g$ is an infinite partial function and $g \subset X$. Since $g$ has infinite intersection with infinitely many things in $\mathscr{A}$, it is clear that $g$ avoids $\mathscr{A}$. Let $a=\operatorname{dom}(g)$ and let $b=\omega \backslash a$. Choose $h \in \mathscr{A}$ and put $f=g \cup h \uparrow b$. Obviously, $f$ is a total function avoiding $\mathscr{A}$. So $f \notin \operatorname{tr}(\mathscr{A})$. Therefore, we may choose an infinite partial function $p \subset f$ such that $\forall h \in \mathscr{A}[|h \cap p|<\omega]$. Clearly, since $\mid p \cap h\lceil b \mid<\omega$, we have that $|p \cap g|=\omega$. Thus, $Y=p \cap g$ is as required.

Definition 3.10. Let $\mathcal{I}$ be a proper non-principal ideal on $\omega$. We will say that $\mathcal{I}$ is a dense ideal if $\forall a \in[\omega]^{\omega} \exists b \in[a]^{\omega}[b \in \mathcal{I}]$.

Lemma 3.11. There is a dense ideal $\mathcal{I}$ on $\omega$ such that whenever $X$ is a subset of $\mathcal{I}$ of size less than $\mathfrak{c}$, there is an infinite set $a \in \mathcal{I}$ such that $\forall x \in X[|a \cap x|<\omega]$.

Proof. Let $\mathscr{A} \subset[\omega]^{\omega}$ be a MAD family of subsets of $\omega$ of size $\mathfrak{c}$, with $\bigcup \mathscr{A}=\omega$. Let $\mathcal{I}$ be the ideal generated by $\mathscr{A}$. It is easily checked that $\mathcal{I}$ is a dense ideal on $\omega$. Now, suppose $X=\left\langle x_{\alpha}: \alpha<\kappa\right\rangle \subset \mathcal{I}$, with $\kappa<\mathfrak{c}$. As $\mathcal{I}$ is generated by $\mathscr{A}$, it is possible to find a set $\mathscr{B} \subset \mathscr{A}$, with $|\mathscr{B}|<\mathfrak{c}$, such that for every $\alpha<\kappa$, there is a finite set $\left\{b_{0}, \ldots, b_{k}\right\} \subset \mathscr{B}$ so that $x_{\alpha} \subset b_{0} \cup \cdots \cup b_{k}$. Since $|\mathscr{A}|=\mathfrak{c}$, we may choose a set $a \in \mathscr{A}$ which is a.d. from everything in $\mathscr{B}$. Now, it is clear that $a$ is the set we are looking for.

Definition 3.12. Let $\mathcal{I}$ be an ideal as in Lemma 3.11 . If $\mathscr{B}$ is a family of infinite partial functions from $\omega$ to $\omega$, we say that $\mathscr{B}$ has domains in $\mathcal{I}$ if $\forall g \in$ $\mathscr{B}[\operatorname{dom}(g) \in \mathcal{I}]$. 
Lemma 3.13. Assume non $(\mathcal{M})=\mathfrak{c}$. Let $\mathcal{I}$ be an ideal as in Lemma 3.11. Let $\mathscr{B}$ be a family of infinite partial functions with domains in $\mathcal{I}$ and let $\mathscr{D} \subset \omega^{\omega}$ be a family of total functions. Suppose that both $\mathscr{B}$ and $\mathscr{D}$ have size less than $\mathfrak{c}$. Let $f \in \omega^{\omega}$ be a.d. from $\mathscr{D}$. There is $h \in \omega^{\omega}$ such that:

(1) $\forall g \in \mathscr{B}[|h \cap g|<\omega]$,

(2) $\forall h^{\prime} \in \mathscr{D}\left[\left|h \cap h^{\prime}\right|<\omega\right]$,

(3) $|h \cap f|=\omega$.

Proof. Let $X=\{\operatorname{dom}(f \cap g): g \in \mathscr{B}\}$. Since $\mathscr{B}$ has domains in $\mathcal{I}$, it follows that $X \subset \mathcal{I}$. By assumption, $|\mathscr{B}|<\mathfrak{c}$. So by Lemma 3.11, we can find an infinite set $a \in \mathcal{I}$ which is a.d. from everything in $X$. Set $p=f \uparrow a$. Since $f$ is assumed to be a.d. from $\mathscr{D}, p$ is also a.d. from $\mathscr{D}$. Moreover, if $g \in \mathscr{B}$, then $\operatorname{dom}(p \cap g) \subset$ $a \cap \operatorname{dom}(f \cap g)$, which is finite. Therefore, $|p \cap g|<\omega$. Now, since non $(\mathcal{M})=c$, there is a total function $h_{0} \in \omega^{\omega}$ which is a.d. from $\mathscr{B} \cup \mathscr{D}$. Let $b=\omega \backslash a$. Set $h=p \cup h_{0}\left\lceil b . h\right.$ satisfies (1) and (2) above because both $p$ and $h_{0}$ are a.d. from $\mathscr{B} \cup \mathscr{D}$. It satisfies (3) because $p$ is an infinite partial function contained in $f$.

Theorem 3.14. Assume non $(\mathcal{M})=\mathfrak{a}=\mathfrak{c}$. There is a $M A D$ family $\mathscr{A} \subset \omega^{\omega}$ with trivial trace 1

Proof. Let $\left\langle f_{\alpha}: \alpha<\mathfrak{c}\right\rangle$ enumerate $\omega^{\omega}$. Let $\mathcal{I}$ be an ideal as in Lemma 3.11, We will construct the MAD family $\mathscr{A}$ by induction, as the union of an increasing sequence of a.d. families. In fact, we will build two sequences $\left\langle\mathscr{A}_{\alpha}: \alpha<\mathfrak{c}\right\rangle$ and $\left\langle\mathscr{B}_{\alpha}: \alpha<\mathfrak{c}\right\rangle$ such that the following hold:

(1) $\mathscr{A}_{\alpha} \subset \omega^{\omega}$ is an a.d. family, with $\left|\mathscr{A}_{\alpha}\right| \leq|\alpha|$.

(2) $\mathscr{B}_{\alpha}$ is a family of infinite partial functions, with $\left|\mathscr{B}_{\alpha}\right| \leq|\alpha|$.

(3) $\forall \alpha<\beta<\mathfrak{c}\left[\mathscr{A}_{\alpha} \subset \mathscr{A}_{\beta} \wedge \mathscr{B}_{\alpha} \subset \mathscr{B}_{\beta}\right]$.

(4) $\mathscr{B}_{\alpha}$ has domains in $\mathcal{I}$.

(5) $\forall h \in \mathscr{A}_{\alpha} \forall g \in \mathscr{B}_{\alpha}[|h \cap g|<\omega]$.

(6) If $f_{\alpha}$ avoids $\bigcup\left\{\mathscr{A}_{\beta}: \beta<\alpha\right\}$, then there is $g \in \mathscr{B}_{\alpha}$ so that $g \subset f_{\alpha}$.

(7) If $f_{\alpha}$ is a.d. from $\bigcup\left\{\mathscr{A}_{\beta}: \beta<\alpha\right\}$, there is $h \in \mathscr{A}_{\alpha}$ so that $\left|h \cap f_{\alpha}\right|=\omega$.

$\mathscr{A}$ will be $\bigcup \mathscr{A}_{\alpha}$. Clauses (1) and (7) ensure that $\mathscr{A}$ is a MAD family in $\omega^{\omega}$. Clauses (5) and (6) ensure that $\mathscr{A}$ has trivial trace. It is easy to see that clause (4) is necessary because if $\mathscr{A}$ is a MAD family with trivial trace, then $\left\{a \in[\omega]^{\omega}\right.$ : $\exists p \in \omega^{a}[p$ is a.d. from $\left.\mathscr{A}]\right\}$ is a proper dense ideal on $\omega$.

Fix $\alpha<\mathfrak{c}$ and suppose that $\left\langle\mathscr{A}_{\beta}: \beta<\alpha\right\rangle$ and $\left\langle\mathscr{B}_{\beta}: \beta<\alpha\right\rangle$ are given to us. Set $\mathscr{B}=\bigcup \mathscr{B}_{\beta}$ and $\mathscr{D}=\bigcup \mathscr{A}_{\beta}$. If $f_{\alpha}$ does not avoid $\mathscr{D}$, then there is nothing to be done. In this case, we simply set $\mathscr{A}_{\alpha}=\mathscr{D}$ and $\mathscr{B}_{\alpha}=\mathscr{B}$. From now on, let us assume that $f_{\alpha}$ avoids $\mathscr{D}$. We will first define $\mathscr{B}_{\alpha}$. Consider $\mathscr{D} \cap f_{\alpha}$. This is an a.d. family on $f_{\alpha}$. Since $|\mathscr{D}|<\mathfrak{c}$, and since, by assumption, $f_{\alpha}$ avoids $\mathscr{D}$, we can find an infinite partial function $p \subset f_{\alpha}$ so that $\forall h \in \mathscr{D}[|p \cap h|<\omega]$. Since $\mathcal{I}$ is a dense ideal, there is an infinite partial function $g_{1} \subset p$ with $\operatorname{dom}\left(g_{1}\right) \in \mathcal{I}$. Now, we define $\mathscr{B}_{\alpha}=\mathscr{B} \cup\left\{g_{1}\right\}$. By our choice of $p$, we have that $\forall h \in \mathscr{D}\left[\left|h \cap g_{1}\right|<\omega\right]$. This completes the definition of $\mathscr{B}_{\alpha}$. We now define $\mathscr{A}_{\alpha}$. We will proceed by cases. Suppose that $f_{\alpha}$ is not a.d. from $\mathscr{D}$. In this case, we may set $\mathscr{A}_{\alpha}=\mathscr{D}$. Note that we have already ensured above that everything in $\mathscr{B}_{\alpha}$ is a.d. from $\mathscr{D}$. So clause (5) will be satisfied. All the other clauses are immediate. Now, let us consider the case

\footnotetext{
${ }^{1}$ An earlier version of this paper claimed to prove this theorem just from the assumption $\mathfrak{a}=\mathfrak{c}$. Brendle pointed out that the proof was implicitly assuming non $(\mathcal{M})=\mathfrak{c}$.
} 
when $f_{\alpha}$ is a.d. from $\mathscr{D}$. $\mathscr{B}_{\alpha}$ is a family of infinite partial functions with domains in $\mathcal{I}$ and its size is less than $\mathfrak{c}$. Also, $\mathscr{D}$ is a family of total functions with $|\mathscr{D}|<\mathfrak{c}$. Therefore, we can apply Lemma 3.13 to find a function $h \in \omega^{\omega}$, which is a.d. from $\mathscr{B}_{\alpha} \cup \mathscr{D}$, with the property that $\left|h \cap f_{\alpha}\right|=\omega$. Now, we can set $\mathscr{A}_{\alpha}=\mathscr{D} \cup\{h\}$. It is easy to see that clauses $(1)-(7)$ are all satisfied, and so we are done.

Observe that if $\mathfrak{a}_{\mathfrak{e}}<\mathfrak{a}$, then any MAD family $\mathscr{A} \subset \omega^{\omega}$ of size $\mathfrak{a}_{\mathfrak{e}}$ will have trivial trace because if $f \in \omega^{\omega}$, then $|\mathscr{A} \cap f|<\mathfrak{a}$. It is unknown if it is consistent to have $\mathfrak{a}_{\mathfrak{e}}<\mathfrak{a}$. We also do not know if the construction in Theorem 3.14 can be carried out under $\mathfrak{a}=\mathfrak{c}$, or even just in ZFC. But we conjecture that the latter is impossible.

Conjecture 3.15. It is consistent with ZFC that every MAD family in $\omega^{\omega}$ has a non-trivial trace.

We point out here that if every ground model has a forcing extension witnessing Conjecture 3.15, then there are no analytic MAD families in $\omega^{\omega}$. This is because the statement expressing the existence of such a family is $\Sigma_{3}^{1}$ and hence relativizes up.

Theorem 3.14 implies that it is consistent to have a MAD family with trivial trace. However, it may still be the case that analytic MAD families cannot have a trivial trace. We investigate this possibility next. We show that analytic MAD families satisfying certain extra combinatorial properties cannot have a trivial trace, and hence, cannot exist. We make use of a partition theorem proved by Taylor [3] and extended by Blass [4].

Theorem 3.16 (Taylor; see 4, Theorem 4). Let $\mathcal{U}$ be a P-point on $\omega$ and let $\mathscr{X} \subset[\omega]^{\omega}$ be an analytic set. There is a set $E \in \mathcal{U}$ and a function $f \in \omega^{\omega}$ such that $\mathscr{X}$ contains all or none of the infinite subsets $F$ of $E$ that satisfy

$$
\forall i, j \in F[i<j \Longrightarrow f(i)<j] .
$$

Convention 3.17. We will apply Theorem 3.16 to an ultrafilter $\mathcal{U}$ on $\omega \times \omega$ and an $\mathscr{X} \subset[\omega \times \omega]^{\omega}$. In order to make sense of the condition $(*)$ in Theorem 3.16 , we must have a well ordering of $\omega \times \omega$ in type $\omega$. Let us arbitrarily choose such an ordering $\prec$.

Lemma 3.18. Let $\mathscr{A} \subset \omega^{\omega}$ be an analytic a.d. family. Let $E \in[\omega \times \omega]^{\omega}$ be a set such that $\exists^{\infty} h \in \mathscr{A}[|h \cap E|=\omega]$. Let $\mathscr{X}=\left\{F \in[\omega \times \omega]^{\omega}: \exists h \in \mathscr{A}[|h \cap F|=\omega]\right\}$. Let $f \in(\omega \times \omega)^{\omega \times \omega}$. There are infinite sets $F_{0}$ and $F_{1}$ in $[E]^{\omega}$ such that $F_{0} \in \mathscr{X}$, $F_{1} \notin \mathscr{X}$ and

$$
\begin{aligned}
& \left(*_{0}\right) \quad \forall\left\langle i_{0}, j_{0}\right\rangle,\left\langle i_{1}, j_{1}\right\rangle \in F_{0}\left[\left\langle i_{0}, j_{0}\right\rangle \prec\left\langle i_{1}, j_{1}\right\rangle \Longrightarrow f\left(\left\langle i_{0}, j_{0}\right\rangle\right) \prec\left\langle i_{1}, j_{1}\right\rangle\right], \\
& \left(*_{1}\right) \quad \forall\left\langle k_{0}, l_{0}\right\rangle,\left\langle k_{1}, l_{1}\right\rangle \in F_{1}\left[\left\langle k_{0}, l_{0}\right\rangle \prec\left\langle k_{1}, l_{1}\right\rangle \Longrightarrow f\left(\left\langle k_{0}, l_{0}\right\rangle\right) \prec\left\langle k_{1}, l_{1}\right\rangle\right] .
\end{aligned}
$$

Proof. Choose $h \in \mathscr{A}$ such that $|h \cap E|=\omega$. We may choose, by recursion, an infinite set $F_{0} \subset h \cap E$ that satisfies $\left(*_{0}\right)$ above. It is clear that $\left|F_{0} \cap h\right|=\omega$, and hence that $F_{0} \in \mathscr{X}$. To get $F_{1}$, we will use Theorem 3.2. Note that $E$ avoids $\mathscr{A}$. So there is $F \in[E]^{\omega}$ such that $F$ is a.d. from $\mathscr{A}$. Once again, we may choose, by recursion, an infinite set $F_{1} \subset F$ that satisfies $\left(*_{1}\right)$ above. It is clear that $F_{1}$ is a.d. from $\mathscr{A}$, and hence that $F_{1} \notin \mathscr{X}$.

Definition 3.19. Let $A$ be a countable set and let $\mathcal{I}$ be a non-principal ideal on $A$. Let $\mathcal{E}=[A]^{\omega} \backslash \mathcal{I}$. We say that $\mathcal{E}$ is a $P$-coideal on $A$ if whenever $E_{0} \supset E_{1} \supset \cdots$ is a sequence of sets in $\mathcal{E}$, there a set $E \in \mathcal{E}$ such that $\forall n \in \omega\left[E \subset^{*} E_{n}\right]$. 
Theorem 3.20. Let $\mathscr{A} \subset \omega^{\omega}$ be an a.d. family. Let $\mathscr{X}=\left\{F \in[\omega \times \omega]^{\omega}: \exists h \in\right.$ $\mathscr{A}[|h \cap F|=\omega]\}$ and let $\mathcal{E}_{0}=\left\{E \in[\omega \times \omega]^{\omega}: \exists^{\infty} h \in \mathscr{A}[|h \cap E|=\omega]\right\}$. If there is a P-coideal $\mathcal{E}$ on $\omega \times \omega$ with $\mathcal{E} \subset \mathcal{E}_{0}$, then $\mathscr{A}$ is not analytic.

Proof. By definition, there is a non-principal ideal $\mathcal{I}$ such that $\mathcal{E}=[\omega \times \omega]^{\omega} \backslash \mathcal{I}$. Let $\mathbb{P}$ be the forcing notion $\mathcal{P}(\omega \times \omega) / \mathcal{I}$. Since $\mathcal{E}$ is a P-coideal, $\mathbb{P}$ is countably closed and hence does not add any reals. Moreover, $\mathbb{P}$ generically adds a P-point $\mathcal{U} \subset \mathcal{E}$. Now, suppose for a contradiction that $\mathscr{A}$ is analytic. Identifying $\omega^{\omega}$ with a $G_{\delta}$ subset of $\mathcal{P}(\omega \times \omega)$ in the natural way makes $\mathscr{A}$ into an analytic subset of $\mathcal{P}(\omega \times \omega)$. This implies that $\mathscr{X}$ is analytic because it has a $\Sigma_{1}^{1}$ definition. As $\mathbb{P}$ does not add any reals, $\mathscr{X}$ is still an analytic set in $\mathbf{V}[\mathcal{U}]$ with the same definition. Now, in $\mathbf{V}[\mathcal{U}]$, we may apply Theorem 3.16 to find a set $E \in \mathcal{U}$ and a function $f \in(\omega \times \omega)^{\omega \times \omega}$ such that $\mathscr{X}$ contains all or none of the infinite subsets $F$ of $E$ that satisfy

$$
\forall\langle i, j\rangle,\langle k, l\rangle \in F[\langle i, j\rangle \prec\langle k, l\rangle \Longrightarrow f(\langle i, j\rangle) \prec\langle k, l\rangle] .
$$

But $\mathbb{P}$ does not add any reals. Therefore, $E$ and $f$ are in the ground model $\mathbf{V}$. Note that $E \in \mathcal{E} \subset \mathcal{E}_{0}$ because $\mathcal{U} \subset \mathcal{E}$. This allows us to apply Lemma 3.18 in $\mathbf{V}$ to find $F_{0}, F_{1} \in[E]^{\omega}$ satisfying $\left(*_{0}\right)$ and $\left(*_{1}\right)$ of Lemma 3.18 with $F_{0} \in \mathscr{X}$ and $F_{1} \notin \mathscr{X}$. But, $F_{0}, F_{1} \in \mathbf{V}[\mathcal{U}]$ still satisfy $\left(*_{0}\right)$ and $\left(*_{1}\right)$ in $\mathbf{V}[\mathcal{U}]$, contradicting the choice of E.

Remark 3.21. If $\mathscr{A}$ is any infinite MAD family in $[\omega]^{\omega}$ and if $\mathcal{E}_{0}=\left\{E \in[\omega]^{\omega}\right.$ : $\left.\exists^{\infty} A \in \mathscr{A}[|E \cap A|=\omega]\right\}$, then Mathias [8] showed that $\mathcal{E}_{0}$ is a P-coideal. It is easy to see that for a MAD family in $\omega^{\omega}, \mathcal{E}_{0}$, as defined in Theorem 3.20, is not necessarily a P-coideal. This is an interesting difference between the two types of MADness.

Theorem 3.20 can be used to deduce further combinatorial constraints on analytic MAD families in $\omega^{\omega}$. Given any a.d. family $\mathscr{A} \subset \omega^{\omega}$, it is natural to define the following ideals on $\omega$, which measure how far $\mathscr{A}$ is from being Van Douwen MAD.

Definition 3.22. Let $\mathscr{A} \subset \omega^{\omega}$ be an a.d. family. We define the ideal

$$
\mathcal{I}_{0}(\mathscr{A})=\left\{a \in \mathcal{P}(\omega): \exists p \in \omega^{a} \forall h \in \mathscr{A}[|p \cap h|<\omega]\right\} .
$$

Given $E \subset \omega \times \omega$, we define $E(n)=\{m \in \omega:\langle n, m\rangle \in E\}$ and $\operatorname{dom}(E)=\{n \in \omega$ : $E(n) \neq 0\}$. We say that $\lim |E(n)|=\infty$ if $\forall k \in \omega \forall^{\infty} n \in \operatorname{dom}(E)[|E(n)|>k]$. We define the ideal $\mathcal{I}_{\infty}(\mathscr{A})$ to be the following set:

$$
\{a \in \mathcal{P}(\omega): \exists E \subset \omega \times \omega[\operatorname{dom}(E)=a \wedge \lim |E(n)|=\infty \wedge \forall h \in \mathscr{A}[|E \cap h|<\omega]]\} .
$$

Notice that $\mathscr{A}$ is a MAD family iff $\omega \notin \mathcal{I}_{0}(\mathscr{A})$ iff $\mathcal{I}_{0}(\mathscr{A}) \neq \mathcal{P}(\omega)$. Observe also that $\mathcal{I}_{\infty}(\mathscr{A}) \subset \mathcal{I}_{0}(\mathscr{A})$. Indeed if $a \in \mathcal{I}_{\infty}(\mathscr{A})$, then there are uncountably many $p \in \omega^{a}$ that are a.d. from $\mathscr{A}$.

Given an analytic a.d. family $\mathscr{A}$, to show that $\mathscr{A}$ is not a MAD family, it suffices to prove that $\mathcal{I}_{0}(\mathscr{A})=\mathcal{P}(\omega)$. While we don't know how to do this, we will show in what follows that $\mathcal{I}_{\infty}(\mathscr{A})$ must be "large" whenever $\mathscr{A} \subset \omega^{\omega}$ is an analytic a.d. family. In particular, we will show that it contains a copy of the ideal $0 \times$ Fin.

Definition 3.23. $0 \times$ Fin $=\{X \subset \omega \times \omega: \forall n \in \omega[|X(n)|<\omega]\}$. 
Lemma 3.24. Let $\mathscr{A} \subset \omega^{\omega}$ be a MAD family. Let

$$
\mathcal{E}=\left\{E \in[\omega \times \omega]^{\omega}: \forall k \in \omega\left[\{n \in \omega:|E(n)|>k\} \notin \mathcal{I}_{\infty}(\mathscr{A})\right]\right\} .
$$

$\mathcal{I}=\mathcal{P}(\omega \times \omega) \backslash \mathcal{E}$ is an ideal on $\omega \times \omega$.

Proof. It is easy to see that $\mathcal{I}$ is closed under subsets. We will check that it is also closed under unions. Fix $E_{0}, E_{1} \in \mathcal{I}$ and suppose, for a contradiction, that $E_{0} \cup E_{1} \in \mathcal{E}$. Observe that $\operatorname{dom}\left(E_{0} \cup E_{1}\right)=\operatorname{dom}\left(E_{0}\right) \cup \operatorname{dom}\left(E_{1}\right)$ and that for all $n \in \omega,\left(E_{0} \cup E_{1}\right)(n)=E_{0}(n) \cup E_{1}(n)$. It follows from our assumption that $E_{0}$ and $E_{1}$ are both in $\mathcal{I}$ and that for some $k \in \omega$, both $\left\{n \in \omega:\left|E_{0}(n)\right|>k\right\}$ and $\left\{n \in \omega:\left|E_{1}(n)\right|>k\right\}$ are elements of $\mathcal{I}_{\infty}(\mathscr{A})$. Since $E_{0} \cup E_{1} \in \mathcal{E},\{n \in$ $\left.\omega:\left|E_{0}(n) \cup E_{1}(n)\right|>2 k\right\} \notin \mathcal{I}_{\infty}(\mathscr{A})$. Therefore, we may choose $n \in \omega$ such that $\left|E_{0}(n)\right| \leq k$ and $\left|E_{1}(n)\right| \leq k$, but that $\left|E_{0}(n) \cup E_{1}(n)\right|>2 k$, a contradiction.

Theorem 3.25. Let $\mathscr{A} \subset \omega^{\omega}$ be a MAD family. If $[\omega]^{\omega} \backslash \mathcal{I}_{\infty}(\mathscr{A})$ is a P-coideal, then $\mathscr{A}$ is not analytic.

Proof. Let $\mathcal{E}_{0}$ be defined as in Theorem 3.20 and $\mathcal{E}$ be as in Lemma 3.24 . Assume that $[\omega]^{\omega} \backslash \mathcal{I}_{\infty}(\mathscr{A})$ is a $\mathrm{P}$-coideal. We must show that $\mathscr{A}$ cannot be analytic. By Theorem 3.20 it is enough to show that $\mathcal{E}$ is a $\mathrm{P}$-coideal and that $\mathcal{E} \subset \mathcal{E}_{0}$. We will first argue that $\mathcal{E}$ is a $\mathrm{P}$-coideal.

Lemma 3.24 tells us that $\mathcal{E}$ is a coideal. Now fix a sequence $E_{0} \supset E_{1} \supset \ldots$ with $E_{i} \in \mathcal{E}$. For each $i$ and $k$, put $a_{k}^{i}=\left\{n \in \omega:\left|E_{i}(n)\right|>k\right\}$. Thus we have $\operatorname{dom}\left(E_{i}\right)=a_{0}^{i} \supset a_{1}^{i} \supset \cdots$. By assumption, no $a_{k}^{i}$ is in $\mathcal{I}_{\infty}(\mathscr{A})$. We also have $a_{k}^{0} \supset a_{k}^{1} \supset \cdots$. Thus, $\left\langle a_{k}^{k}: k \in \omega\right\rangle$ is a decreasing sequence of sets not in $\mathcal{I}_{\infty}(\mathscr{A})$. Since we are assuming that $[\omega]^{\omega} \backslash \mathcal{I}_{\infty}(\mathscr{A})$ is a P-coideal, there is a set $a \notin \mathcal{I}_{\infty}(\mathscr{A})$ such that $a \subset^{*} a_{k}^{k}$, for all $k$. Let us define a set $E \subset \omega \times \omega$ with $\operatorname{dom}(E)=a$ as follows. Let $\left\langle n_{i}: i \in \omega\right\rangle$ enumerate $a$. We may assume that $a \subset a_{0}^{0}$. For each $i \in \omega$, let $l_{i}=\max \left\{k \leq i: n_{i} \in a_{k}^{k}\right\}$. Note that $n_{i} \in a_{l_{i}}^{l_{i}}$ and hence that $\left|E_{l_{i}}\left(n_{i}\right)\right|>l_{i}$. Therefore, we may define $E\left(n_{i}\right)$ to be some (arbitrary) subset of $E_{l_{i}}\left(n_{i}\right)$ of size equal to $l_{i}+1$. We will check that $E$ is as required. Since $a \subset^{*} a_{k}^{k}$ for all $k, \lim l_{i}=\infty$, and therefore, $\lim |E(n)|=\infty$. As $\operatorname{dom}(E)=a \notin \mathcal{I}_{\infty}(\mathscr{A})$, this gives us $E \in \mathcal{E}$. Next, we must check that $E \subset^{*} E_{k}$ for all $k$. Fix $k$. We know that $\forall^{\infty} i \in \omega\left[l_{i} \geq k\right]$. Thus $\forall^{\infty} i \in \omega\left[E\left(n_{i}\right) \subset E_{l_{i}}\left(n_{i}\right) \subset E_{k}\left(n_{i}\right)\right]$. As each $E\left(n_{i}\right)$ is finite, we get that $E \subset^{*} E_{k}$.

Next, we will argue that $\mathcal{E} \subset \mathcal{E}_{0}$. Fix $E \in \mathcal{E}$. We must show that $E$ has infinite intersection with infinitely many members of $\mathscr{A}$. Suppose for a contradiction that there is a finite set $\left\{h_{0}, \ldots, h_{m}\right\} \subset \mathscr{A}$ such that $E$ is a.d. from $\mathscr{A} \backslash\left\{h_{0}, \ldots, h_{m}\right\}$. Thus we have that $E_{0}=E \backslash\left(h_{0} \cup \cdots \cup h_{m}\right)$ is a.d. from $\mathscr{A}$. Notice that for all $n \in \omega, E_{0}(n)=E(n) \backslash\left\{h_{0}(n), \ldots, h_{m}(n)\right\}$. Therefore, $\left|E_{0}(n)\right| \geq|E(n)|-(m+1)$, and so $\{n \in \omega:|E(n)|>k+m+1\} \subset\left\{n \in \omega:\left|E_{0}(n)\right|>k\right\}$ for all $k \in \omega$. Since $E \in \mathcal{E}$, it follows that $\left\{n \in \omega:\left|E_{0}(n)\right|>k\right\} \notin \mathcal{I}_{\infty}(\mathscr{A})$ for all $k \in \omega$. Since we are assuming that $[\omega]^{\omega} \backslash \mathcal{I}_{\infty}(\mathscr{A})$ is a $\mathrm{P}$-coideal, it follows that there is a set $a \notin \mathcal{I}_{\infty}(\mathscr{A})$ such that $a \subset \operatorname{dom}\left(E_{0}\right)$ and for all $k \in \omega, a \subset^{*}\left\{n \in \omega:\left|E_{0}(n)\right|>k\right\}$. Now consider the following subset of $E_{0}: E_{1}=\left\{\langle n, i\rangle \in E_{0}: n \in a\right\}$. Since $E_{0}$ is a.d. from $\mathscr{A}$, $E_{1}$ is a.d. from $\mathscr{A}$. However, $\operatorname{dom}\left(E_{1}\right)=a$ and $\lim \left|E_{1}(n)\right|=\infty$, contradicting $a \notin \mathcal{I}_{\infty}(\mathscr{A})$.

Corollary 3.26. Suppose $\mathscr{A} \subset \omega^{\omega}$ is an analytic MAD family. $\mathcal{I}_{\infty}(\mathscr{A})$ contains a copy of $0 \times$ Fin. This means that there is a partition $\left\{c_{n}: n \in \omega\right\}$ of $\omega$ into countably many infinite pieces such that for any $a \subset \omega$, if $\left|a \cap c_{n}\right|<\omega$ for all $n \in \omega$, then $a \in \mathcal{I}_{\infty}(\mathscr{A})$. 
Proof. By Theorem 3.25 we know that there is a sequence $a_{0} \supset a_{1} \supset \cdots$ of subsets of $\omega$ not in $\mathcal{I}_{\infty}(\mathscr{A})$ such that for any $a \subset \omega$, if $a \subset^{*} a_{n}$ for all $n \in \omega$, then $a \in \mathcal{I}_{\infty}(\mathscr{A})$. We may assume without loss of generality that $a_{0}=\omega$, that $\bigcap a_{n}=0$ and that $a_{n} \backslash a_{n+1}$ is infinite. Put $c_{n}=a_{n} \backslash a_{n+1}$. By our assumptions, $\left\{c_{n}: n \in \omega\right\}$ is a partition of $\omega$ into infinite pieces. Now, suppose $a \subset \omega$ is a.d. from all the $c_{n}$. It is easy to see that for each $n \in \omega, a \backslash a_{n} \subset \bigcup_{m<n}\left(a \cap c_{m}\right)$, which is a finite set. So $\forall n \in \omega\left[a \subset^{*} a_{n}\right]$, whence $a \in \mathcal{I}_{\infty}(\mathscr{A})$.

Conjecture 3.27. If $\mathscr{A} \subset \omega^{\omega}$ is a MAD family, then $\mathscr{A}$ is not analytic.

\section{REFERENCES}

1. Tomek Bartoszyński, Combinatorial aspects of measure and category, Fund. Math. 127 (1987), no. 3, 225-239. MR917147 (88m:04001)

2. Tomek Bartoszyński and Haim Judah, Set theory: On the structure of the real line, A K Peters Ltd., Wellesley, MA, 1995. MR1350295 (96k:03002)

3. James E. Baumgartner and Alan D. Taylor, Partition theorems and ultrafilters, Trans. Amer. Math. Soc. 241 (1978), 283-309. MR0491193 (58:10458)

4. Andreas Blass, Selective ultrafilters and homogeneity, Ann. Pure Appl. Logic 38 (1988), no. 3, 215-255. MR942525 (89h:03081)

5. Jörg Brendle, Otmar Spinas, and Yi Zhang, Uniformity of the meager ideal and maximal cofinitary groups, J. Algebra 232 (2000), no. 1, 209-225. MR.1783921 (2001i:03097)

6. B. Kastermans, J. Steprāns, and Y. Zhang, Analytic and coanalytic families of almost disjoint functions, J. Symbolic Logic 73 (2008), no. 4, 1158-1172. MR.2467209

7. Bart Kastermans, Very mad families, Advances in logic, Contemp. Math., vol. 425, Amer. Math. Soc., Providence, RI, 2007, pp. 105-112. MR2322366 (2008c:03053)

8. A. R. D. Mathias, Happy families, Ann. Math. Logic 12 (1977), no. 1, 59-111. MR0491197 (58:10462)

9. Arnold W. Miller, Arnie Miller's problem list, Set theory of the reals (Ramat Gan, 1991), Israel Math. Conf. Proc., vol. 6, Bar-Ilan Univ., Ramat Gan, 1993, pp. 645-654. MR 1234292 (94m:03073)

10. Yi Zhang, On a class of m.a.d. families, J. Symbolic Logic 64 (1999), no. 2, 737-746. MR1777782 (2001m:03104)

11. - Towards a problem of E. van Douwen and A. Miller, MLQ Math. Log. Q. 45 (1999), no. 2, 183-188. MR.1686175 (2000d:03113)

Department of Mathematics, University of Toronto, Toronto, Ontario, Canada M5S $2 \mathrm{E} 4$

E-mail address: raghavan@math.toronto.edu

$U R L$ : http://www. math.toronto.edu/raghavan 